\title{
Role of Tachyonic Field in Accelerating Universe in Presence of Perfect Fluid
}

\author{
Writambhara Chakraborty ${ }^{1 *}$ and Ujjal Debnath ${ }^{2 \dagger}$ \\ ${ }^{1}$ Department of Mathematics, New Alipore College, \\ New Alipore L Block, Kolkata-700 053, India. \\ ${ }^{2}$ Department of Mathematics, Bengal Engineering and Science University, Shibpur, Howrah-711 103, India.
}

(Dated: January 13, 2019)

\begin{abstract}
Recently tachyonic field has been depicted as dark energy model to represent the present acceleration of the Universe. In this paper we have considered mixture of tachyonic fluid with a perfect fluid. For this purpose we have considered barotropic fluid and Generalized Chaplygin gas(G.C.G.). We have considered a particular form of the scale factor. We have solved the equations of motion to get the exact solutions of the density, tachyonic potential and the tachyonic field. We have introduced a coupling term to show that the interaction decays with time. Also we have shown that the nature of the potentials vary so as the interaction term reduces the potential in both the cases.
\end{abstract}

PACS numbers:

\section{INTRODUCTION}

The observational confirmation about the accelerated expansion of the Universe $[1,2]$ has given rise to a lot of dark energy models [3-6], which are supposed to be the reason behind this present acceleration. This mysterious fluid called dark energy is believed to dominate over the matter content of the Universe by $70 \%$ and to have enough negative pressure as to drive present day acceleration. Most of the dark energy models involve one or more scalar fields with various actions and with or without a scalar field potential [7]. Recently tachyonic field with Lagrangian $\mathcal{L}=-V(T) \sqrt{1+g^{\mu \nu} \partial_{\mu} T \partial_{\nu} T}$ [8] has gained a lot of importance in this respect. The energy-momentum tensor of the tachyon field can be seen as a combination of two fluids, dust with pressure zero and a cosmological constant with $p=-\rho$, thus generating enough negative pressure such as to drive acceleration. Also the tachyon field has a potential which has an unstable maximum at the origin and decays to almost zero as the field goes to infinity. Depending on various forms of this potential following this asymptotic behaviour a lot of works have been carried out on tachyonic dark energy [9, 10], tachyonic dark matter [11, 12] and inflation models [13].

To obtain a suitable evolution of the Universe an interaction is often assumed such that the decay rate should be proportional to the present value of the Hubble parameter for good fit to the expansion history of the Universe as determined by the Supernovae and CMB data [14]. These kind of models describe an energy flow between the components so that no components are conserved separately. An interacting tachyonic-dark matter model has been studied in ref. [15].

In this paper, we consider a model which comprises of a two component mixture. Firstly we consider a mixture of barotropic fluid with tachyonic field without any interaction between them, so that both of them retain their properties separately. Then we consider an energy flow between them by introducing an interaction term which is proportional to the product of the Hubble parameter and the density of the barotropic fluid. We show that the energy flow being considerably high at the beginning falls down noticeably with the evolution of the Universe indicating a more stable situation. Also in both the cases we find the exact solutions for the tachyonic field and the tachyonic potential and show that the tachyonic potential follows the asymptotic behaviour discussed above. Here the tachyonic field behaves as the dark energy component whereas the dust acts as the cold dark matter. Next we consider tachyonic dark matter, the Generalized Chaplygin Gas (GCG) being the dark energy component. GCG, identified by the equation of state (EOS) $p=-B / \rho^{\alpha}$ with $0 \leq \alpha \leq 1$ has been considered as a suitable dark energy model by several authors [16, 17]. Here we consider the mixture of GCG with tachyonic dark matter. Later we have also considered an interaction between these two fluids by introducing a coupling term which is proportional to the product of Hubble constant and the energy density

\footnotetext{
* writam1@yahoo.co.in

$\dagger$ ujjaldebnath@yahoo.com
} 
of the GCG. The coupling function decays with time indicating a strong energy flow at the initial period and weak interaction at later stage implying a stable situation. Like the previous case we have found the exact solution of the tachyonic potential. To keep the observational support of recent acceleration we have considered a particular form of evolution of the Universe here as

$$
a=t^{n}
$$

such that the deceleration parameter reads $q=-\frac{a \ddot{a}}{\dot{a}^{2}}=-\left(1-\frac{1}{n}\right)$, where $a$ is the scale factor. Hence for $n>1$ we always get an accelerated expansion and for $n=1$ we get a constant expansion of the Universe. This kind of recipe has been studied in ref. [12].

The paper is organized as follows:

Section II deals with the field equations of the tachyonic field and Einstein field equations. In sections III and IV we have considered models represented by mixture of tachyonic field with barotropic fluid and GCG respectively. These sections are each subdivided into two parts showing the effect of these models with or without interaction. We have taken some particular values of the parameters and constants for the graphical representation of the tachyonic potential. The paper ends with a short discussion in section V.

\section{TACHYONIC FLUID MODEL AND EINSTEIN FIELD EQUATIONS}

The action for the homogeneous tachyon condensate of string theory in a gravitational background is given by,

$$
S=\int \sqrt{-g} d^{4} x\left[\frac{\mathcal{R}}{16 \pi G}+\mathcal{L}\right]
$$

where $\mathcal{L}$ is the Lagrangian density given by,

$$
\mathcal{L}=-V(T) \sqrt{1+g^{\mu \nu} \partial_{\mu} T \partial_{\nu} T}
$$

where $T$ is the tachyonic field, $V(T)$ is the tachyonic potential and $\mathcal{R}$ is the Ricci Scalar. The energy-momentum tensor for the tachyonic field is,

$$
\begin{gathered}
T_{\mu \nu}=-\frac{2 \delta S}{\sqrt{-g} \delta g^{\mu \nu}}=- \\
V(T) \sqrt{1+g^{\mu \nu} \partial_{\mu} T \partial_{\nu} T} g^{\mu \nu}+V(T) \frac{\partial_{\mu} T \partial_{\nu} T}{\sqrt{1+g^{\mu \nu} \partial_{\mu} T \partial_{\nu} T}} \\
=p_{T} g_{\mu \nu}+\left(p_{T}+\rho_{T}\right) u_{\mu} u_{\nu}
\end{gathered}
$$

where the velocity $u_{\mu}$ is :

$$
u_{\mu}=-\frac{\partial_{\mu} T}{\sqrt{-g^{\mu \nu} \partial_{\mu} T \partial_{\nu} T}}
$$

with $u^{\nu} u_{\nu}=-1$.

The energy density $\rho_{T}$ and the pressure $p_{T}$ of the tachyonic field therefore are,

$$
\rho_{T}=\frac{V(T)}{\sqrt{1-\dot{T}^{2}}}
$$

and

$$
p_{T}=-V(T) \sqrt{1-\dot{T}^{2}}
$$

Hence the EOS parameter of the tachyonic field becomes,

$$
\omega_{T}=\frac{p_{T}}{\rho_{T}}=-\left(1-\dot{T}^{2}\right)
$$


and

$$
p_{T} \rho_{T}=-V^{2}(T)
$$

which represents pure Chaplygin gas if $V(T)$ is constant.

Now the metric of a spatially flat isotropic and homogeneous Universe in FRW model is,

$$
d s^{2}=d t^{2}-a^{2}(t)\left[d r^{2}+r^{2}\left(d \theta^{2}+\sin ^{2} \theta d \phi^{2}\right)\right]
$$

where $a(t)$ is the scale factor.

The Einstein field equations are (choosing $8 \pi G=c=1$ )

$$
3 \frac{\dot{a}^{2}}{a^{2}}=\rho_{t o t}
$$

and

$$
6 \frac{\ddot{a}}{a}=-\left(\rho_{t o t}+3 p_{t o t}\right)
$$

where, $\rho_{\text {tot }}$ and $p_{\text {tot }}$ are the total energy density and the pressure of the Universe.

The energy conservation equation is

$$
\dot{\rho}_{t o t}+3 \frac{\dot{a}}{a}\left(\rho_{t o t}+p_{t o t}\right)=0
$$

\section{MIXTURE OF TACHYONIC DARK ENERGY AND BAROTROPIC FLUID}

Now we consider a two fluid model consisting of tachyonic field and barotropic fluid. The EOS of the barotropic fluid is given by,

$$
p_{b}=\omega_{b} \rho_{b}
$$

where $p_{b}$ and $\rho_{b}$ are the pressure and energy density of the barotropic fluid. Hence the total energy density and pressure are respectively given by,

$$
\rho_{t o t}=\rho_{b}+\rho_{T}
$$

and

$$
p_{\text {tot }}=p_{b}+p_{T}
$$

\section{A. Without Interaction}

First we consider that the two fluids do not interact with each other so that they are conserved separately. Therefore, the conservation equation (13) reduces to,

$$
\dot{\rho}_{T}+3 \frac{\dot{a}}{a}\left(\rho_{T}+p_{T}\right)=0
$$

and

$$
\dot{\rho}_{b}+3 \frac{\dot{a}}{a}\left(\rho_{b}+p_{b}\right)=0
$$




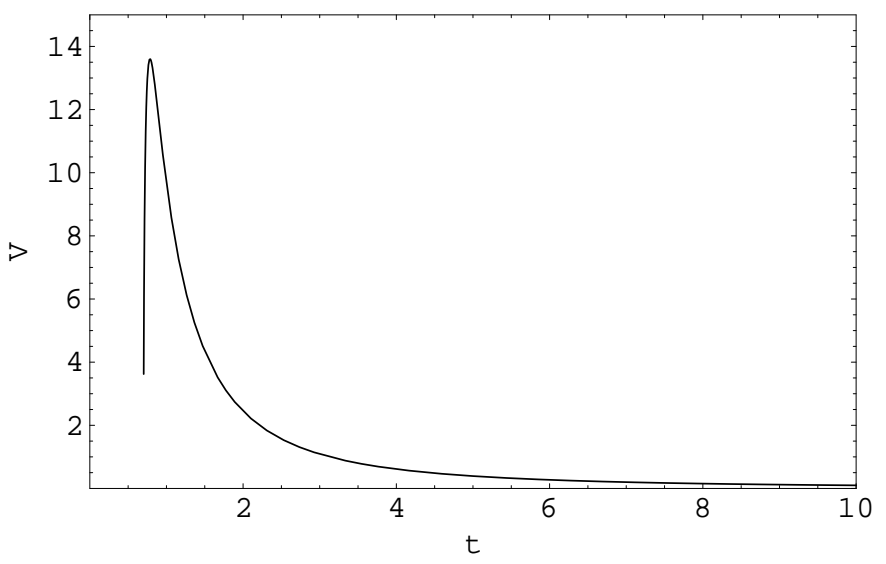

Fig.1

Fig. 1 shows the variation of $V$ against $t$ for $n=2, \rho_{0}=1, \omega_{b}=\frac{1}{2}$.

Equation (18) together with equation (14) give,

$$
\rho_{b}=\rho_{0} a^{-3\left(1+\omega_{b}\right)}
$$

Now, we consider a power law expansion of the scale factor $a(t)$ given by equation (1).

Using (1), equation (19) reduces to,

$$
\rho_{b}=\rho_{0} t^{-3 n\left(1+\omega_{b}\right)}
$$

Also the energy density corresponding to the tachyonic field becomes,

$$
\rho_{T}=\frac{1}{t^{2}}\left[3 n^{2}-\rho_{0} t^{-3 n\left(1+\omega_{b}\right)+2}\right]
$$

Solving the equations the tachyonic field is obtained as,

$$
T=\sqrt{1+\omega_{b}} t \text { Appell } F_{1}\left[\frac{1}{3\left(1+\omega_{b}\right) n-2}, \frac{1}{2},-\frac{1}{2}, 1+\frac{1}{3\left(1+\omega_{b}\right) n-2}, \frac{3 n^{2}}{\rho_{0}} t^{3\left(1+\omega_{b}\right) n-2}, \frac{2 n}{\rho_{0}\left(1+\omega_{b}\right)} t^{3\left(1+\omega_{b}\right) n-2}\right]
$$

where, Appell $F 1\left[a, b_{1}, b_{2}, c, x, y\right]$ is the Appell Hypergeometric function of two variables $x$ and $y$.

Also the potential will be of the form,

$$
V(T)=\sqrt{\frac{3 n^{2}}{t^{2}}-\rho_{0} t^{-3 n\left(1+\omega_{b}\right)}} \sqrt{\frac{3 n^{2}}{t^{2}}-\frac{2 n}{t^{2}}+\omega_{b} \rho_{0} t^{-3 n\left(1+\omega_{b}\right)}}
$$

We can show the graphical representation of the potential against time in figure 1 . We can see that $V \rightarrow 0$ with time, thus retaining the original property of the tachyon potential.

\section{B. With Interaction}

Now we consider an interaction between the tachyonic field and the barotropic fluid by introducing a phenomenological coupling function which is a product of the Hubble parameter and the energy density of the barotropic fluid. Thus there is an energy flow between the two fluids. 


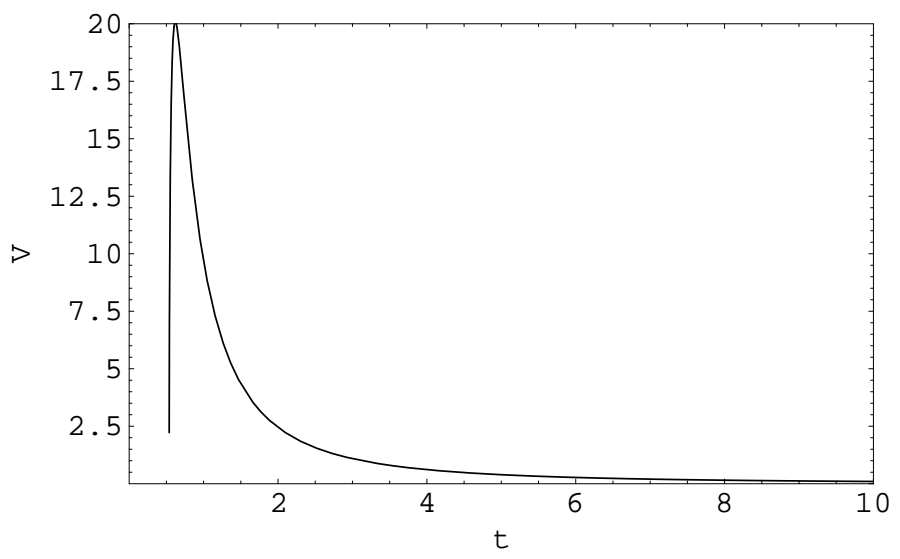

Fig.2

Fig. 2 shows the variation of $V$ against $t$ for $n=2, \rho_{0}=1, \omega_{b}=\frac{1}{2}, \delta=\frac{1}{2}$.

Now the equations of motion corresponding to the tachyonic field and the barotropic fluid are respectively,

$$
\dot{\rho}_{T}+3 \frac{\dot{a}}{a}\left(\rho_{T}+p_{T}\right)=-3 H \delta \rho_{b}
$$

and

$$
\dot{\rho_{b}}+3 \frac{\dot{a}}{a}\left(\rho_{b}+p_{b}\right)=3 H \delta \rho_{b}
$$

where $\delta$ is a coupling constant.

Solving equation (25) with the help of equation (14), we get,

$$
\rho_{b}=\rho_{0} a^{-3\left(1+\omega_{b}-\delta\right)}
$$

Considering the power law expansion (1), we get

$$
\rho_{b}=\rho_{0} t^{-3 n\left(1+\omega_{b}\right)-\delta}
$$

Equation (11) and (27) give,

$$
\rho_{T}=\frac{3 n^{2}}{t^{2}}-\rho_{0} t^{-3 n\left(1+\omega_{b}\right)-\delta}
$$

Solving the equations the tachyonic field is obtained as,

$T=\sqrt{1+\omega_{b}} t$ Appell $F_{1}\left[\frac{1}{3\left(1+\omega_{b}-\delta\right) n-2}, \frac{1}{2},-\frac{1}{2}, 1+\frac{1}{3\left(1+\omega_{b}-\delta\right) n-2}, \frac{3 n^{2}}{\rho_{0}} t^{3\left(1+\omega_{b}-\delta\right) n-2}, \frac{2 n}{\rho_{0}\left(1+\omega_{b}\right)} t^{3\left(1+\omega_{b}-\delta\right) n-2}\right]$

Also the potential will be of the form,

$$
V(T)=\sqrt{\frac{3 n^{2}}{t^{2}}-\rho_{0} t^{-3 n\left(1+\omega_{b}-\delta\right)}} \sqrt{\frac{3 n^{2}}{t^{2}}-\frac{2 n}{t^{2}}+\omega_{b} \rho_{0} t^{-3 n\left(1+\omega_{b}-\delta\right)}}
$$

In this case also $V \rightarrow 0$ with time as shown in the graphical representation of $V$ in figure 2 . 


\section{MIXTURE OF TACHYONIC DARK MATTER AND GENERALIZED CHAPLYGIN GAS}

Now we consider a two fluid model consisting of tachyonic field and G.C.G. The EOS of G.C.G. is given by,

$$
p_{c h}=-B / \rho_{c h}^{\alpha} \quad 0 \leq \alpha \leq 1, B>0 .
$$

where $p_{c h}$ and $\rho_{c h}$ are the pressure and energy density of G.C.G. Hence the total energy density and pressure are respectively given by,

$$
\rho_{t o t}=\rho_{c h}+\rho_{T}
$$

and

$$
p_{t o t}=p_{c h}+p_{T}
$$

\section{A. Without Interaction}

First we consider that the two fluids do not interact with each other so that they are conserved separately. Therefore, the conservation equation (13) reduces to,

$$
\dot{\rho}_{T}+3 \frac{\dot{a}}{a}\left(\rho_{T}+p_{T}\right)=0
$$

and

$$
\dot{\rho}_{c h}+3 \frac{\dot{a}}{a}\left(\rho_{c h}+p_{c h}\right)=0
$$

Equation (35) together with equation (31) give,

$$
\rho_{c h}=\left[B+\frac{\rho_{00}}{a^{3(1+\alpha)}}\right]^{\frac{1}{1+\alpha)}}
$$

Using (1), equation(36) reduces to,

$$
\rho_{c h}=\left[B+\rho_{00} t^{-3 n(1+\alpha)}\right]^{\frac{1}{(1+\alpha)}}
$$

Hence the energy density of the tachyonic fluid is,

$$
\rho_{T}=\frac{3 n^{2}}{t^{2}}-\left[B+\rho_{00} t^{-3 n(1+\alpha)}\right]^{\frac{1}{(1+\alpha)}}
$$

Solving the equations the tachyonic field and the tachyonic potential are obtained as,

$$
T=\int \sqrt{\frac{\frac{2 n}{t^{2}}-\rho_{00} t^{-3 n(1+\alpha)}\left[B+\rho_{00} t^{-3 n(1+\alpha)}\right]^{-\frac{\alpha}{(1+\alpha)}}}{\frac{3 n^{2}}{t^{2}}-\left[B+\rho_{00} t^{-3 n(1+\alpha)}\right]^{\frac{1}{(1+\alpha)}}}} d t
$$

Also the potential will be of the form,

$$
V(T)=\sqrt{\frac{3 n^{2}}{t^{2}}-\left[B+\rho_{00} t^{-3 n(1+\alpha)}\right]^{\frac{1}{(1+\alpha)}}} \sqrt{\frac{3 n^{2}}{t^{2}}-\frac{2 n}{t^{2}}-B\left[B+\rho_{00} t^{-3 n(1+\alpha)}\right]^{-\frac{\alpha}{(1+\alpha)}}}
$$

Like the mixture of tachyonic fluid with barotropic fluid in this case also the potential $V$ starting from a low value increases largely and then decreases to 0 with time as shown in figure 3 . 


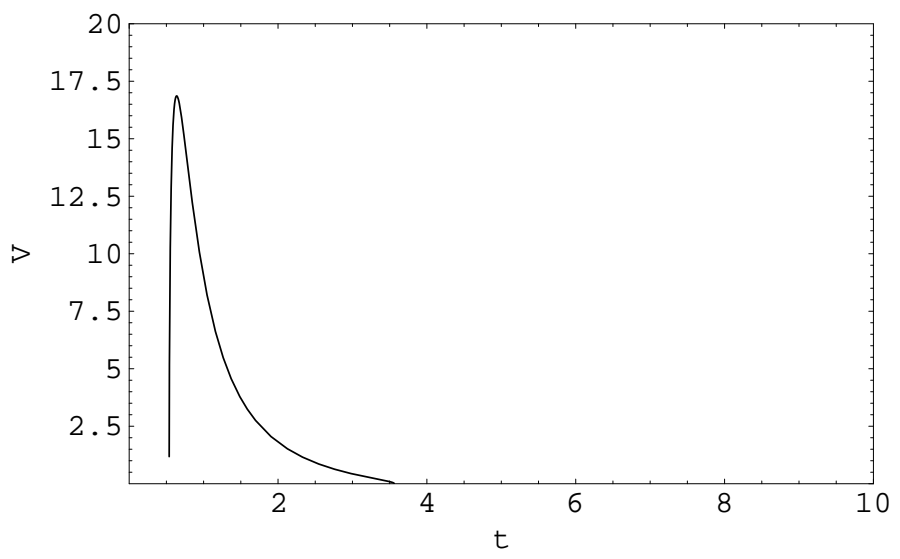

Fig.3

Fig. 3 shows the variation of $V$ against $t$ for $B=\frac{1}{2}, n=2, \rho_{00}=1, \alpha=\frac{1}{2}$.

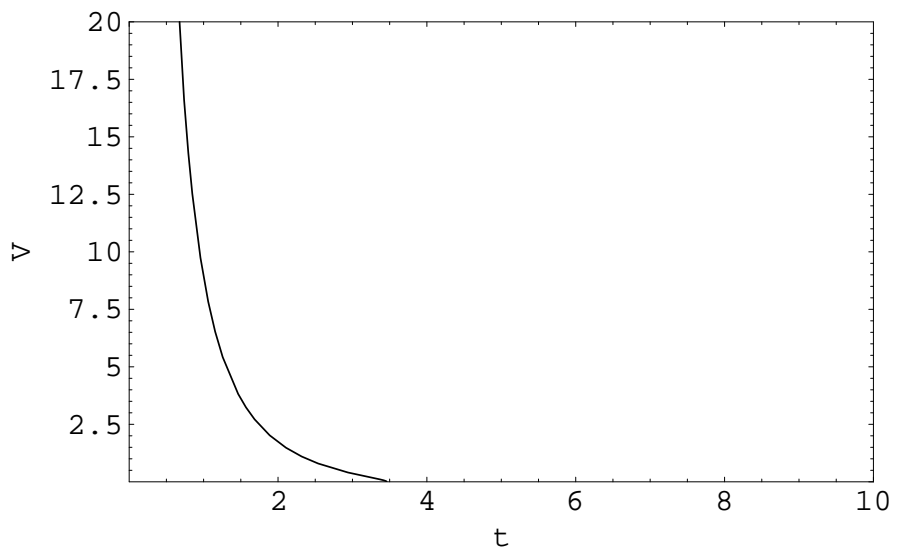

Fig.4

Fig. 4 shows the variation of $V$ against $t$ for $B=\frac{1}{2}, n=2, \rho_{00}=1, \alpha=\frac{1}{2}, \epsilon=\frac{1}{2}$.

\section{B. With Interaction}

Now we consider an interaction between the tachyonic fluid and G.C.G. by phenomenologically introducing an interaction term as a product of the Hubble parameter and the energy density of the Chaplygin gas. Thus there is an energy flow between the two fluids.

Now the equations of motion corresponding to the tachyonic field and G.C.G. are respectively,

$$
\dot{\rho}_{T}+3 \frac{\dot{a}}{a}\left(\rho_{T}+p_{T}\right)=-3 H \epsilon \rho_{c h}
$$

and

$$
\dot{\rho}_{c h}+3 \frac{\dot{a}}{a}\left(\rho_{c h}+p_{c h}\right)=3 H \epsilon \rho_{c h}
$$

where $\epsilon$ is a coupling constant. 
Solving equation (42) with the help of equation (31) and (1), we get,

$$
\rho_{c h}=\left[\frac{B}{1-\epsilon}+\rho_{00} t^{-3 n(1+\alpha)(1-\epsilon)}\right]^{\frac{1}{(1+\alpha)}}
$$

Also the energy density of the tachyonic field will be read as,

$$
\rho_{T}=\frac{3 n^{2}}{t^{2}}-\left[\frac{B}{1-\epsilon}+\rho_{00} t^{-3 n(1+\alpha)(1-\epsilon)}\right]^{\frac{1}{(1+\alpha)}}
$$

Solving the equations the tachyonic field is obtained as,

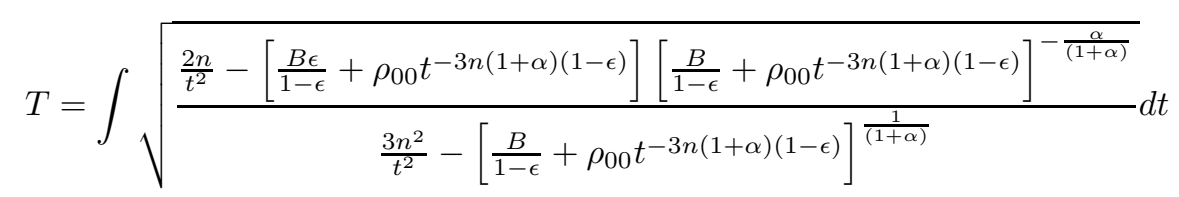

Also the potential will be of the form,

$$
V(T)=\sqrt{\frac{3 n^{2}}{t^{2}}-\left[\frac{B}{1-\epsilon}+\rho_{00} t^{-3 n(1+\alpha)(1-\epsilon)}\right]^{\frac{1}{(1+\alpha)}}} \sqrt{\frac{3 n^{2}}{t^{2}}-\frac{2 n}{t^{2}}-B\left[\frac{B}{1-\epsilon}+\rho_{00} t^{-3 n(1+\alpha)(1-\epsilon)}\right]^{-\frac{\alpha}{(1+\alpha)}}}
$$

In this case the potential starting from a large value tends to 0 (figure 4 ).

\section{DISCUSSION}

We have considered the flat FRW Universe driven by a mixture of tachyonic field and a perfect fluid. We have considered barotropic fluid and Chaplygin gas for this purpose. We have presented accelerating expansion of our Universe due to interaction/without interaction of the mixture of these fluids. We have found the exact solution of the density and potential by considering a power law expansion of the scale factor. We show that these potentials represent the same decaying nature regardless the interaction between the concerned fluids. Since we have considered a power law expansion of the scale factor of the form $a=t^{n}$, we see that for the present acceleration of the Universe to support the observational data we need $n>1$. Now we consider the interaction terms between these fluids. For the mixture of barotropic fluid with tachyonic fluid, we see that the interaction term reduces the potential. Also for the mixture of G.C.G. with tachyonic fluid the interaction parameter $\epsilon$ satisfying $0<\epsilon<1$ so that equation (43) exists for smaller values of $t$. In this case also the interaction reduces the potential. Also if we consider only tachyonic fluid with the power law expansion, we see that the potential (which is obtained to be $V=\frac{3 n^{2}}{t^{2}} \sqrt{1-\frac{2}{3 n}}$ ) is greater than that we get in mixtures. Also the potentials differ in the two cases we have considered. For the mixture with G.C.G. the potential decreases faster than that in case of mixture with barotropic fluid.

\section{Acknowledgement:}

The authors are thankful to IUCAA, India for warm hospitality where part of the work was carried out. Also UD is thankful to UGC, Govt. of India for providing research project grant (No. 32-157/2006(SR)).

\section{References:}

[1] N. A. Bachall, J. P. Ostriker, S. Perlmutter and P. J. Steinhardt, Science 2841481 (1999).

[2] S. J. Perlmutter et al, Astrophys. J. 517565 (1999).

[3] V. Sahni and A. A. Starobinsky, Int. J. Mod. Phys. A 9373 (2000).

[4] P. J. E. Peebles and B. Ratra, Rev. Mod. Phys. 75559 (2003).

[5] T. Padmanabhan, Phys. Rept. 380235 (2003).

[6] E. J. Copeland, M. Sami, S. Tsujikawa, Int. J. Mod. Phys. D 151753 (2006). 
[7] I. Maor and R. Brustein, Phys. Rev. D 67103508 (2003); V. H. Cardenas and S. D. Campo, Phys. Rev. D 69083508 (2004); P.G. Ferreira and M. Joyce, Phys. Rev. D 58023503 (1998).

[8] A. Sen, JHEP 04048 (2002); A. Sen, JHEP 07065 (2002); A. Sen, Mod. Phys. Lett. A 17 1797(2002).

[9] J. S. Bagla, H. K. Jassal and T. Padmanabhan, Phys. Rev. D 67063504 (2003); E. J. Copeland, M.

R. Garousi, M. Sami and S. Tsujikawa, Phys. Rev D 71043003 (2005); G. Calcagni and A. R. Liddle, astro-ph/0606003.

[10] E. J. Copeland, M. Sami and S. Tsujikawa, Int. J. Mod. Phys. D 151753 (2006).

[11] A. DeBenedictis, A. Das and S. Kloster, Gen. Rel. Grav. 362481 (2004); A. Das, S. Gupta, T. D. Saini and S. Kar, Phys. Rev D $\mathbf{7 2} 043528$ (2005).

[12] T. Padmanabhan, Phys. Rev. D 66021301 R (2002).

[13] M. Sami, Mod. Phys. Lett. A 18691 (2003); B. C. Paul and D. Paul, Int. J. Mod. Phys. D 141831 (2005).

[14] M. S. Berger, H. Shojaei, Phys. rev. D 74043530 (2006).

[15] R. Herrera, D. Pavon, W. Zimdahl, gen. Rel. Grav. 362161 (2004).

[16] V. Gorini, A. Kamenshchik and U. Moschella, Phys. Rev. D 67063509 (2003); U. Alam, V. Sahni, T. D. Saini and A.A. Starobinsky, Mon. Not. Roy. Astron. Soc. 344, 1057 (2003).

[17] M. C. Bento, O. Bertolami and A. A. Sen, Phys. Rev. D 66043507 (2002). 